\title{
Editorial: Frontiers in Brain-Based Therapeutic Interventions and Biomarker Research in Child and Adolescent Psychiatry
}

\author{
Paul E. Croarkin ${ }^{1 *}$ and Stephanie H. Ameis ${ }^{2,3,4}$ \\ 'Noninvasive Brain Stimulation Program, Department of Psychiatry and Psychology, Mayo Clinic Depression Center, Mayo \\ Clinic, Rochester, MN, USA, ${ }^{2}$ The Margaret and Wallace McCain Centre for Child, Youth and Family Mental Health, Temerty \\ Centre for Therapeutic Brain Intervention, Slaight Centre for Youth in Transition, Campbell Family Mental Health Research \\ Institute, Centre for Addiction and Mental Health, Toronto, ON, Canada, ${ }^{3}$ Centre for Brain \& Mental Health, The Hospital for \\ Sick Children, Toronto, ON, Canada, ${ }^{4}$ Child \& Youth Mental Health Division, Department of Psychiatry, Faculty of Medicine, \\ University of Toronto, Toronto, ON, Canada
}

Keywords: adolescent, biomarker, child, child and adolescent psychiatry, neuroimaging, non-invasive brain stimulation, pediatric

\section{The Editorial on the Research Topic}

Frontiers in Brain-Based Therapeutic Interventions and Biomarker Research in Child and Adolescent Psychiatry

Childhood psychiatric disorders present challenges given the heterogeneity of presentations, instability of phenotypes, and nascent understanding of neurodevelopment. Recent efforts, such as the National Institute of Mental Health Research Domain Criteria, aim to hone precision medicine approaches for psychiatric disorders (1). Elucidating the ontogeny of psychiatric illnesses and underlying neurobiology is a mandate for advancing modern clinical practice. Recent advances in neuroimaging, preclinical studies, genomics, and non-invasive brain stimulation may soon provide improved monitoring of development in health and disease. These tools also hold great promise for developing biological markers of illness that may be targeted through treatment innovation. This research topic surveys recent developmentally informed clinical neuroscience efforts focused on conditions that affect children and adolescents. Broadly, this includes studies focusing on neurodevelopmental disorders, eating disorders, mood disorders, and treatment innovations.

Specialty section: This article was submitted to Neuroimaging and Stimulation, a section of the journal

Frontiers in Psychiatry

Received: 19 June 2016 Accepted: 27 June 2016

Published: 05 July 2016

Citation:

Croarkin PE and Ameis SH (2016) Editorial: Frontiers in Brain-Based

Therapeutic Interventions and Biomarker Research in Child and

\section{NEURODEVELOPMENTAL DISORDERS}

Recent changes in descriptive diagnostic criteria, such as DSM-5 (2), aim to bridge basic science findings with clinical practice (3). Goldani et al. examine existing literature focused on putative biomarkers of autism spectrum disorder (ASD). Markers of mitochondrial function, oxidative stress, genetic clustering, and inflammation are promising approaches. However, at present, there is insufficient evidence to embed these markers into clinical practice (Goldani et al.). In other efforts to better understand neurobiology in ASD and related neurodevelopmental disorders, Baribeau and Anagnostou review neuroimaging correlates of ASD and schizophrenia. Volumetric changes, cortical thickness differences, and white matter changes in childhood onset schizophrenia (COS) appear to attenuate with age. Impaired local connectivity may also be coupled with amplified long-range connectivity in this condition. Neuroimaging findings in ASD collectively suggest an initial period of brain verdancy followed by dysmorphogenesis in adolescence. Furthermore in ASD, patterns of 
local hyper-connectivity are coupled with impaired long-range neural communication (Baribeau and Anagnostou). Nagamitsu et al. present recent dimensional work with brain single-photon emission computed tomography (SPECT) in participants with attention deficit hyperactivity disorder (ADHD). Children with ADHD, and higher scores on the child behavior checklist-dysregulation profile had significantly increased ${ }^{123}$ I-iomazenil biding in the posterior cingulate cortex. These data suggest that GABAergic inhibitory neurons are involved in the pathophysiology of ADHD (Nagamitsu et al.).

\section{AFFECTIVE DISORDERS}

Recent controversies surrounding the phenotyping of childhood mood disorders underscore the necessity of ongoing work focused on the neurobiological characterization of affective disorders during neurodevelopment (4). Henderson et al. examine white matter microorganization as assessed with diffusion tensor imaging (DTI) in adolescents with depression and healthy controls. Anhedonia and irritability were associated with unique neuroanatomical signatures. This promising early work suggests that prefrontal and limbic tracts are disrupted in depression and unique symptom presentations may have DTI signatures (Henderson et al.). Lee et al. examine brain function of healthy control participants, high-risk offspring, and youth with bipolar disorder by means of a meta-analytic approach. The authors postulated that greater activity in high-risk participants signifies potential compensatory mechanisms, whereas more widespread findings in bipolar patients signified chronic disease processes (Lee et al.). Finally, Walker et al. propose a compelling model for the mood disorder prodrome. These authors posit that early life stress, inflammation, and allosteric load are key contributors to disease burden, disease progression, and neuropathology (Walker et al.).

\section{EATING DISORDERS}

Eating disorders are severely impairing psychiatric illnesses, with high mortality rates, and profound neurobiologic underpinnings (5). Herein, McAdams and Krawczyk describe findings from a study of patients with anorexia, patients with bulimia, and healthy controls. Participants were exposed to social attribution, social identity, and physical identity fMRI tasks. Consistently throughout each region of interest, average activation levels for bulimic participants were greater than the group of patients with anorexia, but less than healthy participants. The authors concluded that patients with eating disorders could have a similar biological substrate in terms of social functioning, yet a distinctive functional characterization is a plausible pursuit for future work (McAdams and Krawczyk). Nagamitsu et al. also present intriguing work focused on developing SPECT biomarkers to guide the treatment of children with anorexia nervosa. In children with anorexia nervosa, decreased ${ }^{123} \mathrm{I}$-iomazenil binding in the anterior cingulate and left parietal cortices was associated with a suboptimal response to treatment. Successful weight restoration was associated with increased relative binding of ${ }^{123} \mathrm{I}$-iomazenil in the posterior cingulate and occipital cortices (Nagamitsu et al.).

\section{TREATMENT INNOVATION IN CHILDREN AND YOUTH}

Transcranial magnetic stimulation (TMS) is a powerful therapeutic and neurophysiological probe. Neurocognitive outcomes are key both in terms of safety and for intervention development, as they may serve as optimal clinical outcome measures in youth $(6,7)$. Wall et al. report on a study in which eighteen depressed adolescents received 30 sessions of $10-\mathrm{Hz}$ rTMS, applied to the left dorsolateral prefrontal cortex. Participants demonstrated improvements in delayed verbal recall and memory. Furthermore, there were no decrements in other neurocognitive dimensions (Wall et al.). Desarkar et al. postulate that imbalances in excitatory and inhibitory neurotransmission could underlie aberrant neuroplasticity in ASD. At the receptor level, this may involve excessive NMDA and deficient GABAmediated neurotransmission. Interventions with high frequency rTMS may have a role in stabilizing dysregulated neuroplasticity in ASD (Desarkar et al.).

In conclusion, the synthesis of neuroscience with child and adolescent psychiatry is yielding important discoveries and new directions for treatment innovation. However, we have yet to make the discoveries necessary to bring neuroscience research into the clinical realm through specific biomarker discovery that could pave the way for precision medicine where biomarkers are profiled in the clinic and individualized treatments are selected to optimize neurodevelopmental trajectories, mitigate the long-term effects of psychiatric illness, and maximize functioning for individuals. Novel research tools, innovative study designs that go beyond the case-control model, longitudinal research that identifies developmental trajectories within heterogeneous conditions, and largescale studies with the power to detect small effects are likely the next frontier in research focused on advancing our understanding of neurobiological underpinnings and developing biologically informed treatments for children and youth with mental illness.

\section{AUTHOR CONTRIBUTIONS}

All authors listed have made substantial, direct, and intellectual contribution to the work and approved it for publication.

\section{ACKNOWLEDGMENTS}

Dr. PC has received financial support from the Brain and Behavior Research Foundation, the Mayo Foundation, and NIMH (grant K23 MH100266). The content of this editorial and research topic is solely the responsibility of the authors and does not necessarily represent the official views of the National Institutes of Health. Dr. SA receives financial support from the CAMH Foundation via the O'Brien Scholarship Fund, the University of Toronto, Faculty of Medicine, Dean's Fund New Staff Grant, and the Ontario Mental Health Foundation. 


\section{REFERENCES}

1. Garvey M, Avenevoli S, Anderson K. The national institute of mental health research domain criteria and clinical research in child and adolescent psychiatry. J Am Acad Child Adolesc Psychiatry (2016) 55(2):93-8. doi:10.1016/j. jaac.2015.11.002

2. American Psychiatric Association. Diagnostic and Statistical Manual of Mental Disorders. 5th ed. Arlington, VA: American Psychiatric Publishing (2013).

3. Lord C, Bishop SL. Recent advances in autism research as reflected in DSM-5 criteria for autism spectrum disorder. Ann Rev Clin Psychol (2015) 11:53-70. doi:10.1146/annurev-clinpsy-032814-112745

4. Wiggins JL, Brotman MA, Adleman NE, Kim P, Oakes AH, Reynolds RC, et al. Neural correlates of irritability in disruptive mood dysregulation and bipolar disorders. Am J Psychiatry (2016). doi:10.1176/appi.ajp.2015.15060833

5. Lutter M, Croghan AE, Cui H. Escaping the golden cage: animal models of eating disorders in the post-diagnostic and statistical manual era. Biol Psychiatry (2016) 79(1):17-24. doi:10.1016/j.biopsych.2015.02.006

6. Krishnan C, Santos L, Peterson MD, Ehinger M. Safety of noninvasive brain stimulation in children and adolescents. Brain Stimul (2015) 8(1):76-87. doi:10.1016/j.brs.2014.10.012
7. Croarkin PE, Daskalakis ZJ. Could repetitive transcranial magnetic stimulation improve neurocognition in early-onset schizophrenia spectrum disorders? JAm Acad Child Adolesc Psychiatry (2012) 51(9):949-51. doi:10.1016/j. jaac.2012.05.012

Conflict of Interest Statement: Dr. PC has received in-kind support for research (supplies and genotyping) from Assurex Health. He has received in-kind support for equipment from Neuronetics. He has received travel support and research support (investigator-initiated trial and serves as a site primary investigator for a multicenter study) from Neuronetics. He has received research support from Pfizer (investigator-initiated grant). Dr. SA has no financial conflicts of interest to declare.

Copyright (c) 2016 Croarkin and Ameis. This is an open-access article distributed under the terms of the Creative Commons Attribution License (CC BY). The use, distribution or reproduction in other forums is permitted, provided the original author(s) or licensor are credited and that the original publication in this journal is cited, in accordance with accepted academic practice. No use, distribution or reproduction is permitted which does not comply with these terms. 